\title{
SLOT-LOADED COMPACT MICROSTRIP ANTENNA FOR DUAL-FREQUENCY OPERATION
}

Sona O. Kundukulam, ${ }^{1}$ Manju Paulson, ${ }^{1}$ C. K. Aanandan, ${ }^{1}$ and F. Mohanan'

${ }^{1}$ Centre for Research in Electromagnetics and Antennas

[lepartment of Electronics

Cochin University of Science and Technology

Cochin 682 022, India

Received 15 June 2001

ABSTRACT: Dual-frequency operation is achieved from a compact microstrip antenna by loading a pair of narrow slots close to its radiating edges. The two frequencies have parallel polarization planes and similar rudiation characteristics. The ratio between the two operating frequencies can be tuned in the range (1.14-1.24), which is much smaller than that of similar designs. The above excellent radiation characteristics are achieved along with an area reduction of $\sim 75 \%$ compared to the standard rectangular patch. (c) 2001 John Wiley \& Sons, Inc. Microwave Opt Technol Lett 31: 379-381, 2001.

Key words: slot loaded; compact; microstrip antenna; dual polarized

\section{INTRODUCTION}

Due to the attractive properties such as light weight, low profile, and low production cost, microstrip antennas are fast replacing conventional antennas. Although rectangular and circular geometries are most commonly used, other geometries having greater size reduction find wide applications in personal communication systems, where the prime concern is compactness. Slot-loaded rectangular patches for attaining dual-frequency operation with the same polarization planes already have been discussed [1-3]. A slot-loaded bow-tie microstrip antenna having a reduced area compared to a standard rectangular patch antenna for dual-frequency operation has been reported in [4]. In this letter, we propose a dual-frequency microstrip antenna design having the same polarization planes using an arrow-shaped microstrip antenna in which a pair of narrow slots are embedded close to the radiating edges of the patch. This antenna has a greater area reduction and a smaller frequency ratio compared to the bow-tie antenna. In a slot-loaded bow-tie microstrip antenna [4], dual-frequency operation is based on the two resonant frequencies of the perturbed $\mathrm{TM}_{10}$ and $\mathrm{TM}_{30}$ modes with a frequency ratio tunable from 2 to 3 . In this proposed design, a lower frequency ratio range is achieved by the excitation of the two adjacent resonant frequencies of the $\mathrm{TM}_{10}$ and $\mathrm{TM}_{\delta 0}$ modes $(1<\delta<2)$ [1]. This makes the antenna more suitable for dual-frequency applications where a lower frequency ratio is required. Experimental results of the dual-frequency characteristics are presented and analyzed.

\section{ANTENNA DESIGN}

The proposed configuration of a dual-frequency arrow-shaped patch antenna is shown in Figure 1. $L$ denotes the length, $W$ the width, $W c d$ the height of the intruding triangle, and $W c p$ the height of the protruding triangle. The structure is etched on a substrate having a thickness $h$ and relative permittivity $\varepsilon_{r}$. A pair of narrow slots having a dimensions of $l_{s} \times w_{s}$ are embedded in the patch parallel to the radiating edges at a distance $s$ from the edges. By choosing suitable values for $W_{c d}$ and $W_{c p}$, two frequencies of the same polarization can be obtained. Keeping $W_{c p}$ a constant and varying $W_{c d}$, the ratio 


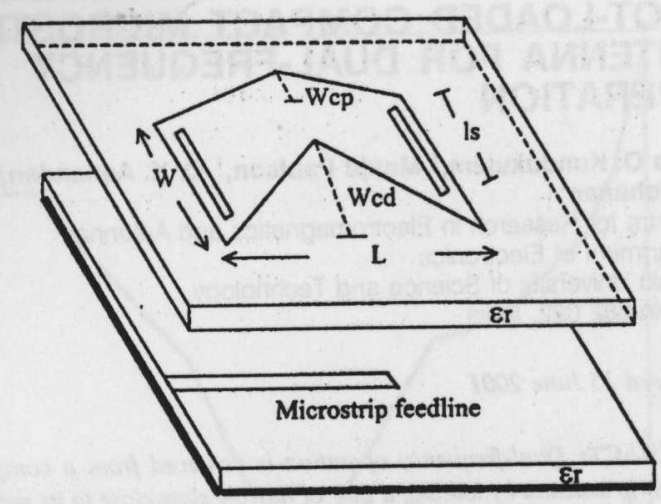

Figure 1 Geometry of the compact microstrip antenna

of the two operating frequencies can be tuned. The antenna is excited by electromagnetic coupling using a $50 \Omega$ microstrip feedline of length $L_{p}$ and width $W_{p}$.

A typical design of the proposed antenna is implemented and investigated. It has dimensions $L=0.06 \mathrm{~m}, W=0.03 \mathrm{~m}$, $W_{c p}=0.01 \mathrm{~m}$, and $W_{c d}=0.02 \mathrm{~m}$, and is fabricated on a substrate of thickness $h=1.6 \mathrm{~mm}$ and dielectric constant $\varepsilon_{r}=4.5$. Slots having dimensions $l_{s}=0.026 \mathrm{~m}$ and $w_{s}=0.002$ $\mathrm{m}$ are placed at a distance $s=0.003 \mathrm{~m}$ from the radiating edges. A good impedance matching of the two operating frequencies can be obtained by using a microstrip feedline of length $L_{p}=0.07 \mathrm{~m}$ and width $W_{p}=0.003 \mathrm{~m}$ etched on a substrate of the same thickness and permittivity, and kept below the antenna to provide electromagnetic coupling.

\section{EXPERIMENTAL RESULTS}

Figure 2 shows the measured return loss against frequency and transmission characteristics. It is found that the frequency ratio changes with $W_{c d}$ of the arrow-shaped patch. The variation of dual frequencies and the frequency ratio with $W_{c d}$ is presented in Table 1 .

The two modes have the same polarization planes and similar radiation patterns. Figures 3 and 4 show the radiation patterns of the antenna for the two frequencies at 0.987 and $1.13 \mathrm{GHz}$, respectively. This design achieves two very close frequencies of the same polarization, and has an area reduction of $77 \%$ for the first frequency and $70 \%$ for the second frequency compared to the standard rectangular patch.

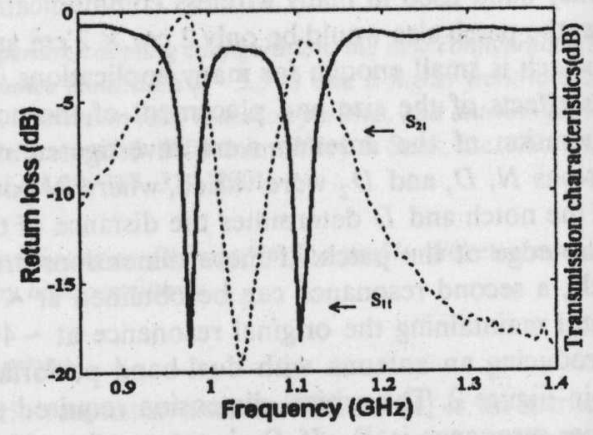

Figure 2 Measured results of the scattering parameters with frequency

TABLE 1 Variation of Dual Frequency with $W_{c d}$ $\left(W_{c p}=0.01 \mathrm{~m}\right)$

\begin{tabular}{lccc}
\hline $\begin{array}{l}W_{c d} \\
(\mathrm{~m})\end{array}$ & $\begin{array}{c}\text { Frequency } \\
f_{1}(\mathrm{GHz})\end{array}$ & $\begin{array}{c}\text { Frequency } \\
f_{2}(\mathrm{GHz})\end{array}$ & $f_{1} / f_{2}$ \\
\hline 0.01 & 0.969 & 1.197 & 1.24 \\
0.015 & 0.974 & 1.197 & 1.23 \\
0.02 & 0.987 & 1.130 & 1.14 \\
0.025 & 0.932 & 1.093 & 1.17 \\
\hline
\end{tabular}
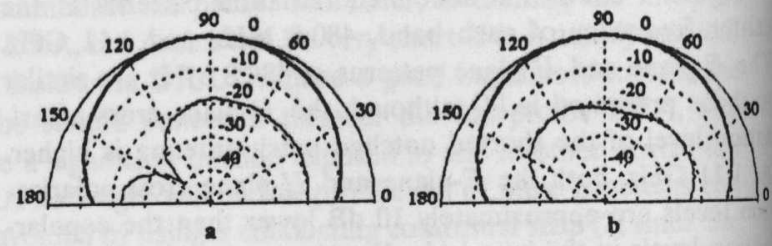

Figure 3 Radiation patterns of the antenna at frequency 0.987 $\mathrm{GHz}$. (a) $H$-plane. (b) $E$-plane. - copolar, -- cross polar

\section{CONCLUSIONS}

A slot-loaded arrow-shaped microstrip patch antenna with dual-frequency operation has been studied experimentally. The two operating frequencies of the proposed design have the same polarization plane and similar radiation characteris. tics. Due to the much-lowered resonant frequency for the present design, the area reduction achieved is $\sim 75 \%$ compared to a standard rectangular patch. Also, the frequencies

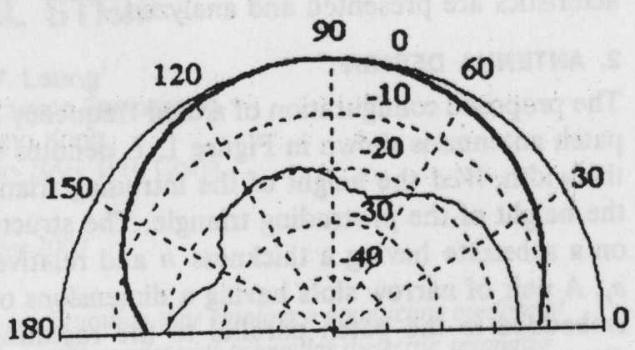

a

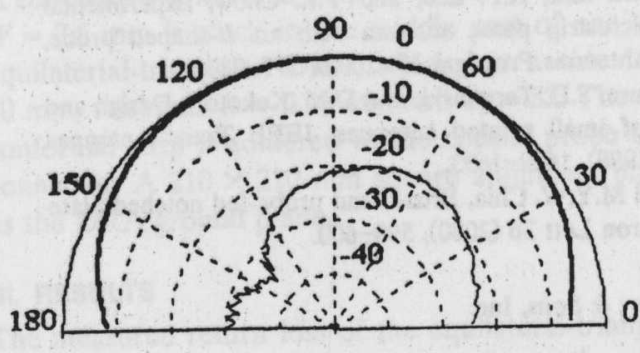

b

Figure 4 Radiation patterns of the antenna at frequency $1.13 \mathrm{GHz}$. (a) $H$-plane. (b) $E$-plane. — copolar. -- cross polar 
obtained are very close to each other, which makes this design more attractive than other slot-loaded patches.

\section{REFERENCES}

1. K.-L. Wong and J.-Y. Sze, Dual-frequency slotted rectangular microstrip antenna, Electron Lett 34 (1998), 1368-1370.

2. J.-H. Lu, Slot-loaded rectangular microstrip antenna for dualfrequency operation, Microwave Opt Technol Lett 24 (2000), 234-237.

3. J.-H. Lu, Single-feed dual-frequency rectangular microstrip antenna with pair of step-slots, Electron Lett 35 (1999), 354-355.

4. K.-L. Wong and W.-S. Chen, Slot-loaded bow-tie microstrip antenna for dual-frequency operation, Electron Lett 34 (1998), 1713-1714.

(c) 2001 John Wiley \& Sons, Inc. 